\title{
Erratum: "Photochromic Reactions in Silver Nanocomposites with a Fractal Structure and Their Comparative Characteristics" [Technical Physics 48 (6), 749 (2003)]
}

\author{
S. V. Karpov, A. K. Popov, and V. V. Slabko
}

"Asymmetric broadening (width)" and "symmetric broadening (width)" should be replaced by "inhomogeneous broadening (width)" and "homogeneous broadening (width)," respectively, throughout the text. 